Bol. Soc. Geol. Mexicana XXXV: 41-45, 1974.

\title{
Participación del Geólogo en el Desarrollo de Nuestro País.
}

\author{
E. López Ramos \\ Superintendencia de Geología Regional. PEMEX.
}

\author{
EL INGENIERO GEOLOGO.-INICIACION Y \\ DESARROLLO DE LA CARRERA.
}

La Carrera de geólogo fué fundada en la Facultad de Ciencias en la UNAM, el año de 1935. En el año de 1939 se establece la carrera de Geólogo en la Escuela Nacional de Ingenieros con 4 años $y$ en 1949, la de Ingenero Geólogo, con duración de 5 años, habiéndose recibido el primer alumno con este título, el año de 1953. En la Escuela Superior de Ingeniería y Arquitectura, I.P.N., se inicia en el año de 1939, con 3 años de duración lectiva, las carreras de Ing. Geólogo Petrolero, la de Geólogo en 1943 con 4 años y en el año de 1959 egresaron por primera vez alumnos de la carrera de Ing. Geólogo, de 5 años de duración, la cual incluyó básicamente las materias de matemáticas superiores, hidráulica y física que también llevaban los ingenieros civiles y que preparaba al profesionista para abordar problemas como mecánica de suelos, ingeniería de minas y geología aplicada a la ingeniería civil en los proyectos y obras de puentes, carreteras, cimentación de edificios, etc. También se ampliaron los cursos de geología en materias que no se impartían en las carreras de 4 años, así el profesionista egresado contaba con un panorama muy amplio de las ciencias geológícas, las cuales son las únicas que requíeren una gama considerable de conocimientos aparte de las matemáticas, como son la química, física, biología, cibernética, etc.

En la Universidad de San Luis Potosí (Escuela de Ingeniería), en el mes de Febrero de 1960, es fun- dada la carrera de Ingeniero Geólogo con 4 años de duración, y posteriormente, en 1973, a 5 años.

\section{NUMERO DE EGRESADOS}

Los primeros geólogos egresados fueron los de la Facultad de Ciencias. UNAM en el año de 1938 y en la ESIA fueron en el año de 1942, con 5 elementos, los que se incrementan a un promedio de 75 geólogos en los últimos 3 años.

De acuerdo con la Fig. 1 el total de geólogos e Ings. geólogos egresados hasta 1974, es el siguiente:

Esc. Sup. de Ing. y Arq. INP

Esc. Nac. de Ing. Fac. de Ing. UNAM

Esc. de Geología.-Universidad S.L.P.

Total

1079.

Admitiendo el total de 280 elementos en las Cías. Mineras, (Tabla No. 1), se verá que no se tiene conocimiento de la actividad de 110 geólogos.

Actualmente hay inscritos más de 700 alumnos en la carrera de Ingeniero-Geólogo y se estima que en los próximos años aumentará el promedio anual de Ings. Geólogos, no solamente en el I.P.N., sino en el resto de las escuelas.

\section{CAMPO DE ACTIVIDAD}

Entre los años de 1950 y 1962. Petróleos Mexicanos empleaba alrededor del $70 \%$ de los egresados de la carrera, actualmente la mísma empresa solo emplea unos 240 elementos del total de geólogos de Méxíco, 


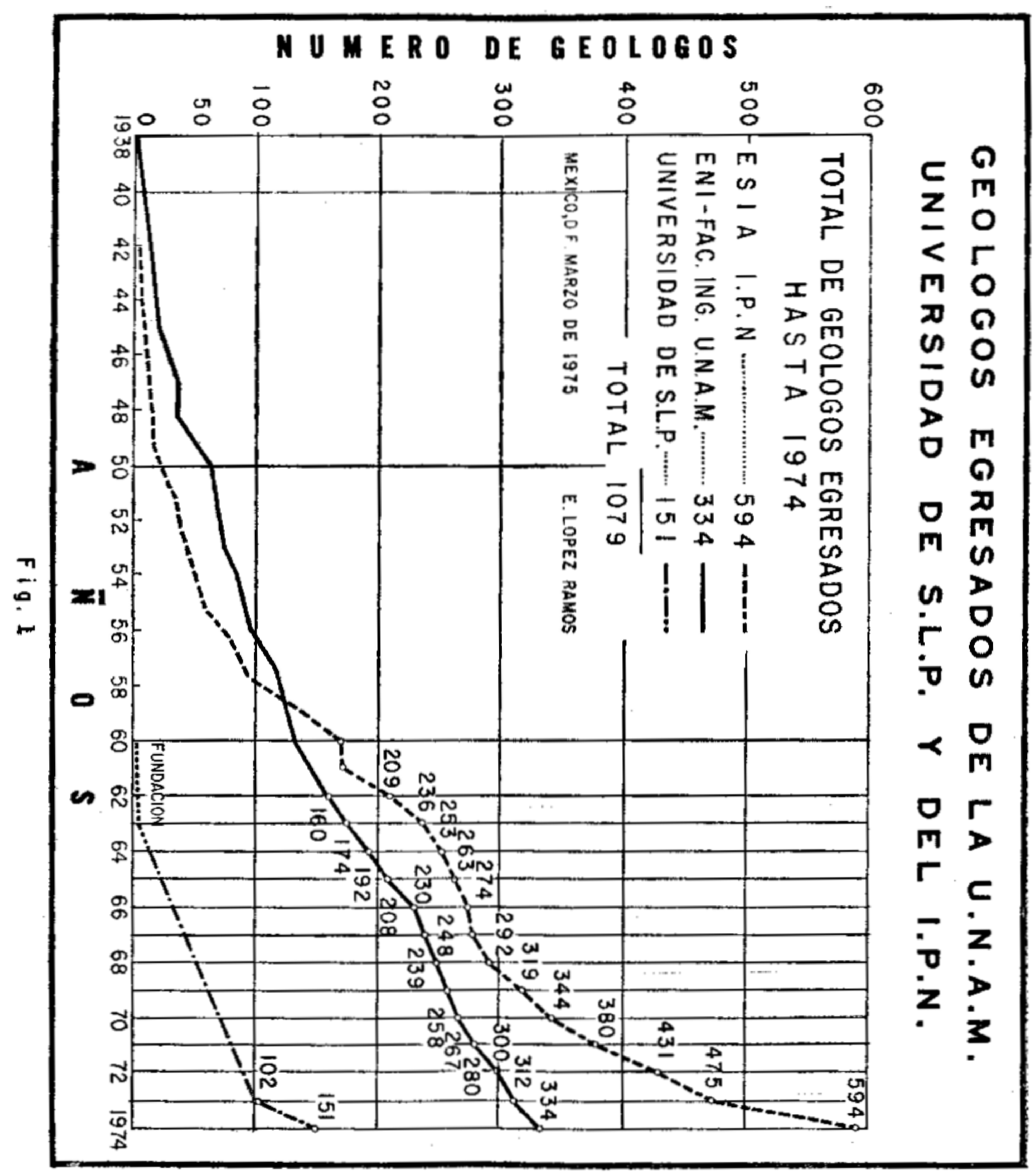


que se estima en 1079 , o sea poco menos del $25 \%$. Otras dependencias y empresas que se citan a continuación y en orden de importancia, emplean a los geólogos ordinariamente y son las siguientes:

\section{Consejo de Recursos Naturales No Renovables (CRNNR)}

\section{Compañías Mineras}

Secretaría de Recursos Hidráulicos

Comisión de Estudios del Territorio Nacional y Planeación (CETENAL).

Instituto de Geología UNAM

Comisión Federal de Electricidad.

Comisión Nacional de Energía Nuclear.

Secretaría de Obras Públicas (Depto. de Geología). Instituto Mexicano del Petróleo y

Consultores en Genera1 (véase Tabla No. 1).

Con el propósito de desglosar el amplísimo campo de actividad del geólogo, a continuación se enumeran las principales ramas a las cuales podría dedicarse:

Como Fotogeólogo.-Para levantar totalmente la carta fotogramétrica y geológica de la República para su uso en todas las actividades científicas, tanto de planeación como de construcción, evaluación de recursos naturales etc.

Como Edafólogos e Hidrólogos.-Se trabaja actualmente en estudios de suelos y levantamiento del nivel freático de las cuencas hidrológicas, aunque es palpable que falta personal para estos estudios, pues es evidente que no han cubierto toda la República.

Como Geólogos Petroleros.-La demanda de este tipo de geólogos no será apreciable en los próximos años debido a que Petróleos Mexicanos cuenta con el plantel más numeroso, antiguo y competente. Todos ellos laboran en dicha Institución y en el Instituto Mexicano del Petróleo. De no ampliarse sustancialmente los programas de exploración, el número de geólogos en los próximos diez años sólo se incrementará en unos 150 .

Como Geólogos Mineros.-En la rama de Geología de Minas, es donde el papel del geólogo no ha sido decisivo en muchos aspectos, ya que las dependencias gubernamentales los han utilizado sólo para valorar las reservas y en algunos casos, desde luego, en búsqueda de yacimientos minerales. Es posible que un papel más activo de geólogo minero conjuntamente con el ingeniero de minas, sea de grandísima importancia en el incremento, tanto de las reservas, como de la producción de minerales. La demanda de cuando menos unos 400 geólogos mineros en los próximos 8 años deberá ser cubierta.

Como Geólogos Civiles.-Como se indicó inicialmente, es ésta una de las especializaciones más amplia a la que puede dedicarse el geólogo y cuyo campo prácticamente está virgen. Si la expansión del país está en razón directa de las construcciones de carreteras, presas, edificios, que constituyen la infraestructura, no hay duda de que la demanda de especialidades se incrementará notablemente.

\section{PREPARACION PROFESIONAL.}

El tema anterior es muy controvertido por varias razones: Tanto los planes de estudio académicos, como el desarrollo de las prácticas de campo y laboratorios, bibliotecas, estaban bien planeadas originalmente, inclusive en forma muy superior a los conocimientos de Bachelor in Sciences de los E.U.A y ligeramente inferior a los Master in Sciences, del mismo país; sin embargo, la preparación de nuestros egresados deja mucho que desear si consideramos los siguientes factores:

1.-Exceso de población estudiantil, lo que ocasiona que los elementos de que se cuenta para su aprendizaje, tales como aulas, maestros y laboratorios, sean insuficientes.

2.-Preparación inapropiada en los estudios de Prevocacional y Vocacional (Secundaria-Preparatoria).

3.-Interrupciones continuas de las labores escolares, que ocasionan que los programas de clase no se cumplan.

4.-En los primeros 15 años de la carrera de geólogo, se daba gran interés a la geología petrolera, pero debido a la saturación de geólogos en la industria, actualmente se hace una preparación más general incluyendo cierta tendencia por la geología de minas y geohidrología.

Como consecuencia de lo anterior, se sugiere lo siguiente:

1.-Como no es posible asegurar trabajo a generaciones tan considerables, de más de 150 geólogos anualmente. deberá limitarse la inscripción de alumnos con exámenes de admisión para seleccionar a los más destacados.

2.-Esta labor de planeación deberá afirmarse con el cumplimiento del reglamento escolar relativo a 
que se obtengan solo alumnos regulares en bimestres profesionales del 3 al 10.

3.-Elevar el nivel profesional de los licenciados en geología, con el establecimiento a nivel nacional de las maestrías y doctorados en ciencias geológicas.

\section{UBICACION EN EL CAMPO PROFESIONAL}

Debe indicarse enfáticamente, que la fuerza de trabajo del geólogo egresado solo es aprovechada en un $75 \%$, que se realiza principalmente en el campo y sólo un porcentaje mínimo ocupa puestos directivos de regular importancia en empresas y prácticamente carece de proyección nacional en grandes corporaciones o bien como geólogo consultor.

Conviene indicar que actualmente se cuenta con egresados de altos estudios de grados de maestro (42 elementos), Doctor en Ciencias (18 elementos), este número es muy bajo, dado el número de años de fundación de las escuelas de Geología.

Participación de actividades de Planeación, Dirección y ejecución relativas a su profesión. En el inciso anterior se explicó la posición de nuestro geólogo en el campo profesional, la cual es relativamente precaria respecto a dirección y consecuentemente a la planeación, que es privativa de los ejecutivos o bien superintendentes de obras.

Con honrosas excepciones, el geólogo ha sido utilizado únicamente como fuerza de trabajo en el campo y es oportuno desde ahora impartir una nueva filosofía a nuestros geólogos en el sentido de que próximamente serán ellos los directores y consultores de grandes empresas. desde luego, deberán prepararse con estudios profesionales completos, además de cursos de post grado, idiomas, capacitación en administración de empresas y otras actividades.

\section{OPORTUNIDADES DE TRABAJO DE LA ESPECIALIDAD EN EL PAIS.}

Al tratar el campo de actividad de nuestros geólogos, se mencionan las ramas de trabajo en las que podrían desarrollarse y en las que predomina la geología de minas, la geohidrología, fotogeología y geología civil. Además hay una actividad que no se ha tomado en cuenta, que es la de maestro en las ciencias de la tierra, actualmente hay más de 100 profesores de geología que tienen esta actividad como marginal a su verdadera profesión de geólogo. El crear profesores de tiempo completo con salarios atractivos, sería de gran importancia y alivio al cuadro de maestros de geología.

Según información reciente, hay más de 700 estudiantes de geología en las 3 escuelas donde se imparte la carrera y desde luego es alarmante el problema que se avecina de proporcionar trabajo a estos egresados, por lo que se impone la planeación inmediata para controlar y regularizar el número y calidad de egresados.

\section{CONCLUSIONES}

Aparentemente no hay problema en la actualidad para acomodar en empleos a los egresados cada año, pero si los habrá, cuando el número actual de geólogos se duplique o triplique. Debe tomarse en cuenta que un número mayor de geólogos traerá consigo problemas de profesores, equipo y laboratorios, que en caso de no resolverse, tendrá consecuencias en el futuro de los egresados, pues ya anticipadamente sabemos, que su preparación académica será incompleta, lo cual podrá lamentarse posteriormente, cuando existan decenas de muchachos que soliciten un empleo en el cual dedicaron largos años de estudio.

Surge entonces la interrogante, ¿qué pasos deben darse en forma inmediata, para resolver, o tratar de resolver, el problema que se avecina de la gran oferta de empleos de la carrera de geólogo?, ya en el Boletín 1-2, Vol. III, Ene-Feb., 1974, del Colegio de Ingenieros de Minas, Metalurgistas, Petroleros y Geólogos de México, se habían propuesto algunas alternativas, respecto a la posible limitación de inscripciones para la carrera de Ingeniero Geólogo, desafortunadamente, tanto la política de nuestras autoridades educativas, como de las instituciones y escuelas, no han previsto hnsta ahora, un control sobre la inscripción limitada, debido a que la demanda de geólogos es hasta ahora superior a la oferta, situación que cambiará a partir de 1975, cuando la oferta sea superior.

Según se avecinan las necesidades de una mejor preparación. para hacer frente a las necesidades de las diferentes industrias extractivas, será necesario precisamente que el grado ordinario para un mejor empleo, sea el de maestría en lugar de la licenciatura y en una etapa superior, el Doctorado; este último tendrá que establecerse en forma permanente en los próximos 10 años. 
TABLA No. 1

TOTAL DE GEOLOGOS EN MEXICO

\begin{tabular}{|c|c|c|c|c|c|}
\hline & Pasantes & $\begin{array}{l}\text { Con Licen- } \\
\text { ciatura }\end{array}$ & Maestría & Doctorado & Total \\
\hline Petróleos Mexicanos & 22 & 203 & 8 & 0 & 233 \\
\hline $\begin{array}{l}\text { Inst. Mexicano del Petróleo } \\
* \text { incluye } 2 \text { Biólogos }\end{array}$ & - & 21 & 9 & 6 & $36^{*}$ \\
\hline C.R.N.N.R. & 35 & 65 & 2 & 3 & 105 \\
\hline Inst. Nac. de E. Nuclear & 6 & 27 & 3 & --- & 36 \\
\hline Inst. de Geología, UNAM & - & 19 & 7 & 9 & 35 \\
\hline \multicolumn{4}{|l|}{ Direc. de Obras Hidráulicas } & - & 4 \\
\hline $\begin{array}{l}\text { CETENAL } \\
\text { Secretaría de Recursos Hidráulicos }\end{array}$ & 32 & $\begin{array}{l}19 \\
32\end{array}$ & $\begin{array}{l}1 \\
9\end{array}$ & - & $\begin{array}{l}52 \\
76\end{array}$ \\
\hline Comisión Federal de Electricidad & - & 11 & 2 & - & 13 \\
\hline Sría. de Obras Públicas & 4 & 5 & 1 & - & 10 \\
\hline Sría de Salubridad y Asistencia & & & & & 8 \\
\hline Sría. de Agricultura & & & & & 7 \\
\hline $\begin{array}{l}\text { Cía. Mex. de Aerofoto } \\
\text { Cías. Mineras (Asarco, Peñoles } \\
\quad \text { y Mineros Particulares) }\end{array}$ & - & 6 & 1 & - & $\begin{array}{c}10 \\
280^{\star}\end{array}$ \\
\hline Consultores & & & & & 15 \\
\hline Varios (Incluye los que estudiaron e & el extranjero). & & & & 32 \\
\hline \multirow[t]{2}{*}{ Fallecidos } & & & & & 12 \\
\hline & & & & & 8 \\
\hline Total: & 134 & $774^{* *}$ & 43 & 18 & 969 \\
\hline
\end{tabular}

México. D. F., Marzo de 1975

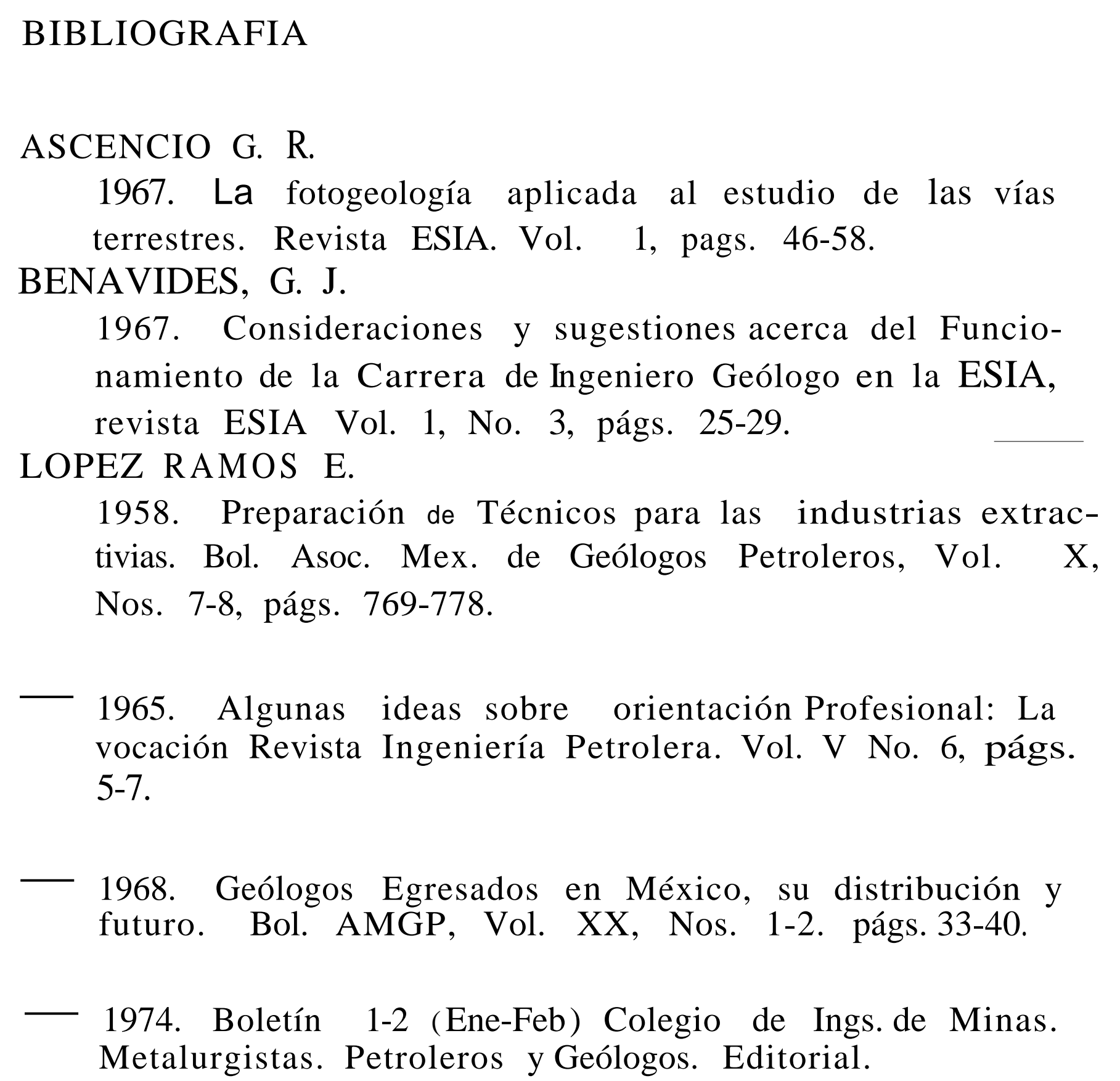

